\title{
Scientific advances in fire modelling and its integration in a forest fire decision system
}

\author{
L. Ferragut ${ }^{1}$, S. Monedero ${ }^{2}$, M. I. Asensio ${ }^{1} \&$ J. Ramírez ${ }^{2}$ \\ ${ }^{1}$ Instituto Universitario de Fisica Fundamental y Matematicas, \\ Salamanca, Spain \\ ${ }^{2}$ Tecnosylva, sl. Leon, Spain
}

\begin{abstract}
At present, there exists a wide range of fire behavior models and studies aiming to simulate fire. Nevertheless there is still an important gap between the scientific research in this field and its practical use by fire fighting agencies. In this work, we intend to narrow this gap by the introduction of a fire combustion model integrated within an easy to use GIS environment aiming to give real and practical solutions for fire fighting agencies. The present paper focuses on the combustion model fire behavior implementation carried out in this GIS platform. Combustion models describe the evolution of physical quantities inside of the vegetation, considered as a porous medium, and as a consequence provide the evolution of the fire front. Between combustion models we can distinguish two groups: 1) Complex models, where general conservation laws are considered and several phases, solid phase and gas phase with different temperatures, two layers model, etc. 2) Simplified models, where in general an average media is considered with average magnitudes. Usually only one temperature is considered and only one phase (the other phase is parameterized). The simplified models are more realistic than models based on cellular automata, geometric models and other empirical models, but allow faster computations than complex models. The models in this work takes into account the main mechanisms in fire spread: Water content, Radiation, Wind and topography effects. Concerning the final GIS product. It is part of the European Eurorisk-Preview project (Geo-information services for risk management on a European level), and offers the following useful services: 1) Access to long lasting layer information: High definition fuel types, terrain, fire breaks (rivers, roads, highways, etc). 2) Access to real time web based updated layer information: Meteorological wind, moisture content of the terrain. 3) Rothermel based fire simulator. 4) Combustion fire behavior model. 5) High Definition Wind Model. All integrated within an easy to use environment, 3D mapping, and easy inputs and outputs in all standard formats.
\end{abstract}

Keywords: forest fires simplified models, moisture, multivalued operator, non local radiation, local radiation, GIS implementation. 


\section{Introduction}

Combustion models are based on balance laws of physics and intend to keep track of the real physical variables involved in a fire. They are usually posed on a bidimensional domain (for real time computations this is mandatory) representing the vegetation layer which is considered as a porous medium where the main variables of fire develop. Coupled to these equations through certain parameters, several important physical effects like wind, moisture, tilt or radiation are evaluated.

The general deduction and simplification of the initial three dimensional differential equations is complex as can be seen in [2] and [3]. Consequently equations are usually simply posed following plausible physical arguments. Despite of the relatively wide range of different approximations to the problem the basic structure of the differential equations to be solved usually conserve a common structure. The following adimensional equations may represent a simple but realistic model.

$$
\begin{gathered}
\frac{\partial e}{\partial t}+\mathbf{w} \cdot \nabla e-\left(J * u^{4}-u^{4}\right)+\alpha u=A f(u, y) \\
e \in G(u) \\
\frac{\partial y}{\partial t}=-f(u, y)
\end{gathered}
$$

Here $e$ is the non-dimensional enthalpy, $u$ is the non-dimensional temperature and $y$ is the non-dimensional mass fraction of fuel.

The non-dimensional enthalpy $e$ introduced accounts for moisture effects, and is an element of a multivalued maximal monotone operator $G$, given by:

$$
G(u)= \begin{cases}u & \text { if } u<u_{v} \\ {\left[u_{v}, u_{v}+\lambda_{v}\right]} & \text { if } u=u_{v} \\ u+\lambda_{v} & \text { if } u_{v}<u<u_{p} \\ {\left[u_{p}+\lambda_{v}, u_{p}+\lambda_{v}+\lambda_{p}\right]} & \text { if } u=u_{p} \\ u+\lambda_{v}+\lambda_{p} & \text { if } u>u_{p}\end{cases}
$$

where $u_{v}$ and $u_{p}$, are the non-dimensional evaporation temperature of the water and the non-dimensional pyrolysis temperature of the solid fuel, respectively. The quantities $\lambda_{v}$ and $\lambda_{p}$ are the non-dimensional evaporation heat and pyrolysis heat.

The right hand side functions $f$ follow an Arrhenius type law and modelize on one side the loss of fuel and on the other the energy gain due to combustion. The term $\alpha u$ represents the energy lost by convection in the vertical direction.

If the medium is optically thick the non local radiation $-\left(J * u^{4}-u^{4}\right)$ could be approximated by a non linear diffusion term plus an additional convection term if the kernel $J(\cdot)$ is non symmetric. This asymmetry is usually due to wind and 
surface tilt. Clearly using a Taylor expansion

$$
\begin{aligned}
\left(J * u^{4}\right)(x)-u^{4} & =\int J(x-y) u^{4}(y) d y-u^{4}(x) \\
& =-\left(\int J(z) z d z\right)\left(u^{4}\right)^{\prime}(x)+\left(\int J(z) z^{2} d z\right)\left(u^{4}\right)^{\prime \prime}(x)+\cdots
\end{aligned}
$$

If the kernel $J($.$) is symmetric then$

$$
-\left(J * u^{4}-u^{4}\right) \approx-\left(\int J(z) z^{2} d z\right)\left(4 u^{3} u^{\prime}\right)^{\prime}(x)
$$

Thermal radiation is nearly always the dominant mode of heat transfer in the vicinity of a large fire source. Furthermore, it is usually accepted that radiation form the flames above the vegetable layer is not only the dominant radiative contribution, but also the main mechanism of fire spread [5]. The numerical treatment of radiation is computationally expensive since it is highly nonlinear and a three dimensional phenomena. So radiative models are restricted to approximated schemes. In this paper we present two simplified models for the thermal radiation: a local radiation diffusive model, and a non local radiation model.

Wind is an important hazard parameter in forest fire and thus a convenient local windfield should be generated. In the present examples the model developed in [6] is implemented.

\section{Model with local radiation}

In this first model we propose a simplified 2-D model where radiation is approximated by a non-linear diffusive term. The convective term takes into account the effect of the meteorological wind and the slope of the terrain. The non-dimensional equations are derived from the conservation law of energy and mass fraction using the Frank-Kamenetskii change of variables.

Let $Q=\left[0, l_{x}\right] \times\left[0, l_{y}\right] \subset \Re^{2}$ a rectangle representing the projection of the surface where the fire takes place, big enough so the fire does not arrive to the boundary and therefore Dirichlet boundary conditions can be considered.

$$
\begin{array}{rlrl}
\partial_{t} e+\mathbf{w} \cdot \nabla e-\nabla(K(u) \nabla u)+\alpha u & =A f(u, y), & & \text { in } S \quad t \in\left(0, t_{\max }\right), \\
e \in G(u), & & \text { in } S \quad t \in\left(0, t_{\max }\right), \\
\partial_{t} y & =-B f(u, y), & & \text { in } S \quad t \in\left(0, t_{\max }\right),
\end{array}
$$

where again $e$ is the non-dimensional enthalpy, $u$ is the non-dimensional temperature and $y$ is the non-dimensional mass fraction of fuel.

The non linear function $K(u)=\kappa(1+\epsilon u)^{3}+1$, in the diffusive term represents the local radiation. The parameter $K$ depends on the Stefan-Boltzmann constant, the optical path length for radiation, the thermal conductivity and the ambient temperature. The parameter $\epsilon$ depends on the activation energy, the universal constant of gases and the ambient temperature. 
The function $f(u, y)=\left(u>u_{p c}\right) y e^{\frac{u}{1+\varepsilon u}}$ is given by an Arrhenius type law where $u_{p c}$ is the non dimensional temperature at which the process changes from endothermic to exothermic, and the logic expressions is equal to 1 if the expression is true and 0 if the expression is false. We write the pre-exponential factors $A$ and $B$, out of the function $f$, in order to simplify notation. Notice that the preexponential factor $B$ in 6 depends on the reaction heat.For more details about how to derive this model, its solution and applications see [8].

\section{Model with non local radiation}

Let $S$ be a surface defined by the mapping

$$
\begin{gathered}
S: Q \longmapsto \mathfrak{R}^{3} \\
x, y \longrightarrow(x, y, h(x, y))
\end{gathered}
$$

representing the part of the terrain where the propagation of a fire can take place. We will assume that vegetation can be represented by a given fuel load together with a moisture content. Besides we will assume that the height of the flames in a particular fire are known and bounded by $H$. In order to take into account some three dimensional effects, and particularly the radiation from the flames above the surface $S$, we will consider the following three dimensional domain

$$
D=\left\{(x, y, z) \in \mathfrak{R}^{3} \quad(x, y) \in Q, \quad h(x, y)<z<h(x, y)+H\right\}
$$

This model considers the energy and mass conservation equations in the surface $S$, and the radiation equation in $D$.

$$
\begin{array}{rcc}
\partial_{t} e+\alpha u=r & \text { in } S & t \in\left(0, t_{\max }\right) \\
e \in G(u) & \text { in } S & t \in\left(0, t_{\max }\right) \\
\partial_{t} y=-B f(u, y), & \text { in } S & t \in\left(0, t_{\max }\right),
\end{array}
$$

Again the function $f$ follows an Arrhenius type law $f(u, y)=\left(u>u_{p}\right)(y>$ $\left.y_{e}\right) y \exp (-\gamma /(1+u))$ where $y_{e}$ is the mass fraction lower bound of extinction.

The right hand side of equation (7) describes the thermal radiation reaching the surface $S$ from the flame above the layer. The intensity is defined as the radiation energy passing through an area per unit time, per unit of projected area and per unit of solid angle. The projected area is formed by taking the area that the energy is passing through and projecting it normal to the direction of travel. The unit elemental solid angle is centered about the direction of travel and has its origin at the area element.

After adimensionalization, the radiation equations in the direction $\Omega$ can be written as

$$
\begin{gathered}
\Omega . \nabla i+a^{*} i=\delta\left(1+u_{g}\right)^{4} \quad \text { in } D \\
i=0 \quad \text { on } \partial D \cap\{\mathbf{x} ; \Omega . \mathbf{n}<0\}
\end{gathered}
$$


where $i$ is the non-dimensional radiation intensity, $a^{*}$ is the non-dimensional absorbtion coefficient, $u_{g}$ is the non dimensional flame temperature and $\delta$ depends on the Stefan-Boltzmann constant. The right hand side represents the total emissive power of a blackbody. The incident energy at a point $\mathbf{x}(x, y, h(x, y))$ of the surface $S$ due to radiation from the flame above the surface per unit time and per unit area will be obtained summing up the contribution of all directions $\Omega$, that is

$$
r(\mathbf{x})=\int_{\omega=0}^{2 \pi} i(\mathbf{x}, \Omega) \Omega . \mathbf{n} d \omega
$$

where we have only considered the hemisphere above the fuel layer.

For more details about how derive this model, its solution and applications see [4].

\section{GIS}

All real time forest fire models share the final goal of providing useful tools for forest fire agencies. Empirical models like Farsite whose relevance and usefulness in fire management and safety is completely out of question, has some relevant drawbacks. Firstly, only users with the proper fire behavior training and experience may use it. Secondly, it does not include a high definition wind model, even when wind plays a dominant roll in fire spread, and finally, users must have the support of a geographic information system (GIS) because the tool requires spatial landscape information and fuel type information to run. Obviously combustion models also have their drawbacks as the research field is still very active and there is neither a clear final set of equations nor a final set of parameters to be used, and much work is still ahead for these models to be openly accepted by fire agencies.

Nevertheless we intend to take advantage of the synergy among different models by means of Geographical Information Systems, which are without a doubt the perfect framework tool for fire agencies and fire simulators. They allow an easy overlaying of data layers, from vegetation data or fire contour to transportation networks, fire fighting resources or private information layers. There is no need of long format conversion and we may take advantage of the highly relevant synergy created by implementing in the same framework different fire behaviors tools or wind models.

This GIS integration is a part of PREVIEW. European VI Framework Programme Integrated Project intending to setup the next generation of services for disaster management support, within the GMES initiative. A cluster of experienced organizations within Europe is preparing the basis of this new generation of services, compiled in a single portfolio covering all scales at pan-European level, built from final user needs and taking into account all previous efforts in the area.

The main structure of the tool is given in figure 1. There are three differentiated modules and two main key features. The modules are an implementation of Rothermel's model through Esris's tools, the non local radiation physical model presented here, and a High Definition Wind Field $(H D W F)$. The key features are 


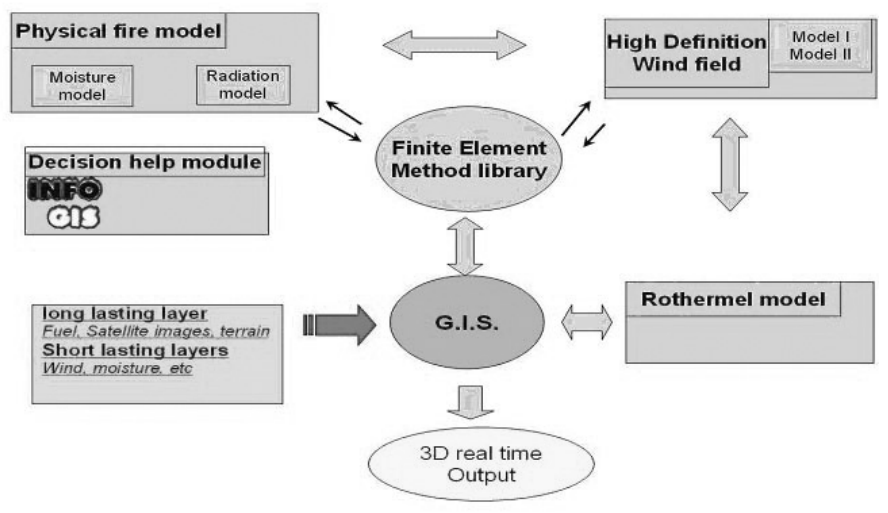

Figure 1: General logical structure of the tool.

the Finite Element Library which solves both, the physically based and the high definition wind model, and the GIS system which serves as the integrating tool for the rest of modules. The numerical resolution of the equations is based on the Finite Element Method with and without mesh refinement. Since ArcGIS has limited computing capabilities and does not allow a numerical treatment of this extent, the implementation is based on the Finite Element Library Neptuno++ developed by Prof. Ferragut [7]. Apart from these, some additional services may be implemented. One of these services is the Decision support system INFOGIS ${ }^{T M}$ developed by Tecnosylva which analyzes the emergency situation, gives immediate information about the distribution of fire fighting means and helps in the following up process of the fire. The layers used are provided by Tecnosylva. The present definition and extent of the pre-rasterized layers are 25 meters covering all Spain. The fuel type classification follows the NFFL types and is given in vector format. A classification of firebreaks has been added so we can distinguish rivers, roads, etc. At present the meteorological wind needed for the HDWF and the moisture content is introduced by hand but the integration with real time meteo GIS data forecasting via GeoRRSS web services is being developed, so the main task for the final user will be to set the ignition point (line or polygon) in the map.

Figure 2 left shows an example of a fire front together the HDWF associated with the simulation. Figure 2 Right shows an example of the 3D output using google earth. This output may be easily extended to other 3D softwares. Figure 3 Left and Right show the outputs of the physical model. The output consists on a temperature layer (figure 3 Left) and a fuel layer (figure 3 Right). The temperature layer is an adimensionalized layer from which the fire front is easily identified. Notice that the physical model does not only provide the fire front, but the overall extension of the fire. The fuel layer is the amount of vegetation fuel left to be used. In the present example shown in figure 5 Right, different fuel types were given different amounts of fuel. 

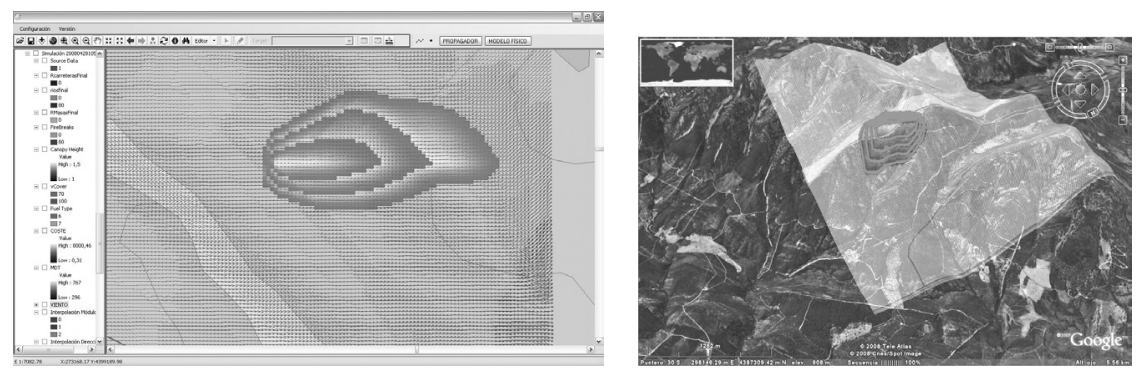

Figure 2: Left: Windfield and fire perimeter. Right: 3D google earth output.
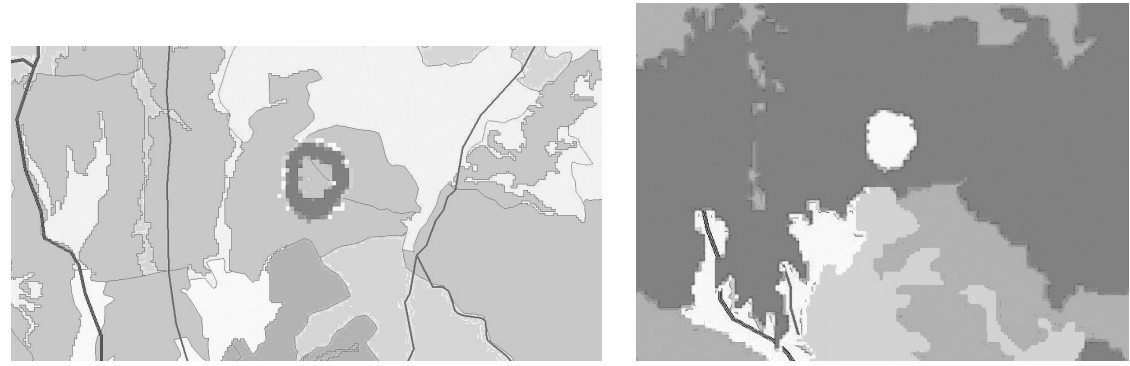

Figure 3: Left: Temperature layer. Right: Fuel layer.

The natural synergy among the tools here exposed and the GIS environment should be highlighted. Standard GIS features like editing layers, saving projects, etc, may allow particularly useful possibilities for fire fighting. We may for example easily change vegetation types in a map, edit firebreaks in a forest, load private information layers over the same view, like cadastral maps or previously made risk maps, etc.

\section{Conclusions}

In the present work two different $2 \mathrm{D}$ combustion models of fire spread aiming to simulate fire in close to real time have been introduced. The models are solved by the FEM. The main innovation concerns the way radiation and moisture content are treated. The former through a characteristic method which lowers the computational time and the latter through a multivalued operator. It is important to mention that even though at present the research in combustion models may still be very open, and there is not a clear set of equations nor a clear set of parameters to be used, the presented approaches do not confine to the presented models and may be easily implemented in other combustion models.

Together with the combustion models, a general operational software thought to be used in actual fire fighting activities and based on a GIS system has also been introduced. The software also includes a high definition windfield solved by the FEM, and a Rothermel type fire propagator. The synergy and practical usefulness 
generated by the implementation of the different models in one GIS tool should be highlighted.

\section{Acknowledgements}

Spanish Ministry of Education and Science through the project CGL2007-65680C03-03/CLI. European Commission through the EU FP6 project Eurorisk-Preview.

\section{References}

[1] R.C. Rothermel. A mathematical model for predicting fire spread in wildland fuels. General Technical Report INT-115, USDA Forest Service, Intermountain Forest and Range Experiment Station, 1972.

[2] Margerit J, Séro Guillaume O. Modelling forest fires. Part II: reduction to two-dimensional models and simulation of propagation, Int. J. Heat and Mass Transfer 45:1723-1737, 2002.

[3] Margerit J, Séro Guillaume O. Modelling forest fires. Part I: A complete set of equations derived by extended irreversible thermodynamics. Int. J. Heat and Mass Transfer ; 45:1723-1737, 2002.

[4] L. Ferragut, M.I. Asensio, S. Monedero. Modelling radiation and moisture content in fire spread. Communications in Numerical Methods in Engineering 23, 819-833, (2007).

[5] Cox G. Combustion Fundamentals of Fire. Academic Press, London, 1995.

[6] M.I. Asensio, L. Ferragut, J. Simon, “Aconvection model for fire spread simulation”, Applied Mathematics Letters 18, 673-677,(2005).

[7] L. Ferragut http://web.usal.es/ferragut/,

[8] M. I Asensio, L. Ferragut. On a wildland fire model with radiation Int. J. Num. Methods Eng. 54, 137-157,(2002). 